\title{
How to design a cartographic continuum to help users to navigate between two topographic styles?
}

\author{
Jérémie Ory, ${ }^{\mathrm{a}}$ Guillaume Touya, ${ }^{\mathrm{a}}$ Charlotte Hoarau, ${ }^{\mathrm{a}}$ and Sidonie Christophe ${ }^{\mathrm{a}}$ \\ ${ }^{a}$ Univ. Paris-Est, LASTIG COGIT, IGN, ENSG, F-94160 Saint-Mandé, France ; firstname.surname@ign.fr
}

\begin{abstract}
Geoportals and geovisualization tools provide to users various cartographic abstractions that describe differently a geographical space. Our purpose is to be able to design cartographic continuums, i.e. a set of in-between maps al-lowing users to navigate between two topographic styles. This paper addresses the problem of the interpolation be-tween two topographic abstractions with different styles. We detail our approach in two steps. Firstly, we set-up a comparison in order to identify which structural elements of a cartographic abstraction should be interpolated. Secondly, we propose an approach based on two design methods for maps interpolation.
\end{abstract}

Keywords: geovisualization, cartographic continuum, topographic style, interpolation, map design, perception and cognition

\section{Introduction}

The last two decades, next to the traditional maps producers, i.e. National Mapping Agencies from different countries, new maps producers gradually emerged: Google Maps, OpenStreetMap (OSM), Stamen, MapBox and so on. This diversification of maps producers naturally leads to the multiplication of cartographic abstractions with a different cartographic content and a particular visual appearance defined as a topographic style (Kent and Vujakovic, 2009; Jolivet, 2009; Beconyte, 2011; Christophe, 2012; Ory et al., 2015). These various cartographic abstractions are provided to users in geovisualization applications in order to respond to theirs uses (Ruas, 2006; Bucher et al., 2007; Mericksay, 2011; Griffin and Fabrikant, 2012; Lafay et al., 2015; amongst others). According to their uses, needs and preferences, users switch from a cartographic abstraction to another. In this context, one of the major challenges consists in proposing innovative visualization methods to smooth and improve the navigation between two different cartographic abstractions with various styles on a geoportal.

We propose to design cartographic continuums, i.e. a set of in-between maps enabling users to navigate between two topographic styles. An important issue of this work is to define a method to design a continuum composed of in-between cartographic abstractions mixing two topographic styles. In this paper, we aim at identifying which structural elements of a cartographic abstraction should be interpolated and how to design them to get a relevant cartographic continuum.

In the following, Section 2 provides the related work. In Section 3, we detail our approach to identify which structural elements of a map should be interpolated and how to design them in a cartographic continuum to navigate be-tween two topographic styles.

\section{Related work}

Designing cartographic continuums between two topographic styles relate to several parts of map design: data matching, generalization, legend specifications and so on. This section describes some research that we believe to be closely related to our problems.

\subsection{Characterizing discontinuities between various cartographic abstractions}

To optimize the multi-scale navigation, Dumont et al. (2015) identify different sources of user disorientation such as symbolization and content discontinuities. The Figure 1 illustrates the symbolization discontinuities between three various topographic abstractions at the same scale depicting the same geographical space: Zürich in Switzer-land. According to the topographic map visualized, the salient objects seem different. On Google Maps representation (A) the hydrologic surfaces seems the most salient objects. On OpenStreetMap (B) representation the road net-work seems the most salient objects and on the Swisstopo representation (C) the buildings seems the most salient objects. These symbolization disparities are based on the legend specification choices, such as color, line width, texture and so on (Jolivet, 2009, Kent and Vujakovic, 2009, Christophe, 2012, Ory et al., 2013).

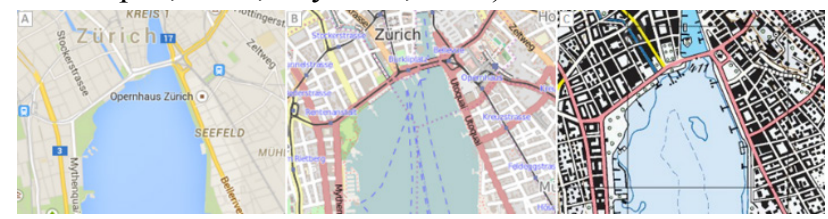

Fig. 1. Three various topographic abstractions at the same scale: (A) Google Maps, (B) OpenStreetMap, (C) Swisstopo (Dumont et al., 2015). 


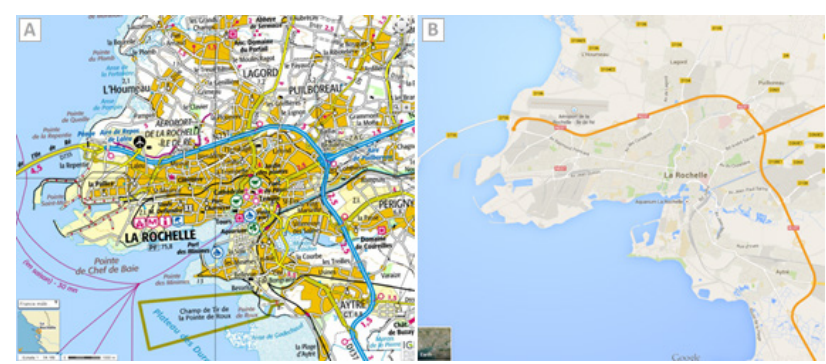

Fig. 2. Difference of content quantity between maps from IGNFrance (A) and Google Maps (B) - (Dumont et al., 2015).

The Figure 2 illustrates the content discontinuities between two topographic abstractions, IGN-France (A) and Google Maps (B), which depicted the same geographical space: La Rochelle in France. A simplification of the level of details from the IGN-France to the Google Maps implies different map contents for the same displayed scale.

These symbolization and content discontinuities should be managed by a relevant approach to design a cartographic continuum between two topographic abstractions.

\subsection{Characterizing a topographic style}

Ory et al. (2015) formalize the concept of a topographic style as a cartographic family with a visual signature composed of graphic signs perceived and recognized by users. From the results of a user study, Ory (2016) characterize two levels of a visual signature for IGNFrance topographic style. The Figure 3 illustrates two levels of a visual signature. The graphic signs content in the level (A) are both perceived and memorized by participants. The graphic sign content in level (B) are only perceived by participants. Ory (2016) relies on these two levels of visual signature to produce topographic maps with a recognizable style by users.

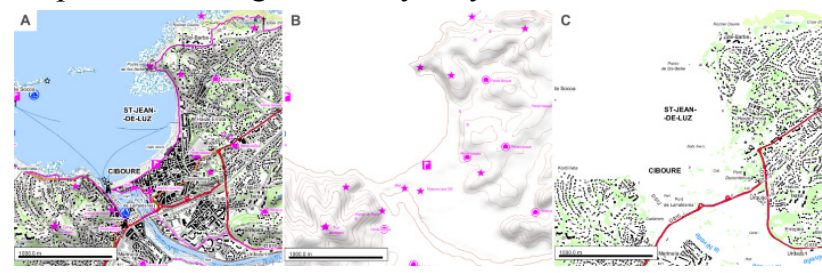

Fig. 3. (A) 1:25000 IGN-France topographic map, (B) 1st level of visual signature, (C) 2nd level of visual signature - (Ory, 2016).

The knowledge on the graphic signs recognized by users and the visual signature of a topographic style should be managed to design a cartographic continuum between two topographic styles.

\subsection{Design method of the interpolation between vector data and orthoimage}

Hoarau and Christophe (2016) propose a global rendering method to mix efficiently topographic vector data and orthoimages. This method is based on natural appearance extraction, color and texture interpolation, and the control of the realism and abstraction levels, allowing to design a cartographic continuum composed of several in-between representations. The Figure 4 illustrates a cartographic continuum between a vector data set with IGN-France topographic style and an orthoimage. The legibility of cartographic objects is preserved all along the continuum, providing efficient covisualizations of the two representations to the user. The cartographic continuum allows users to smoothly navigate between two different representations of a geographical space, or to stop on a legible and effective in-between representation.

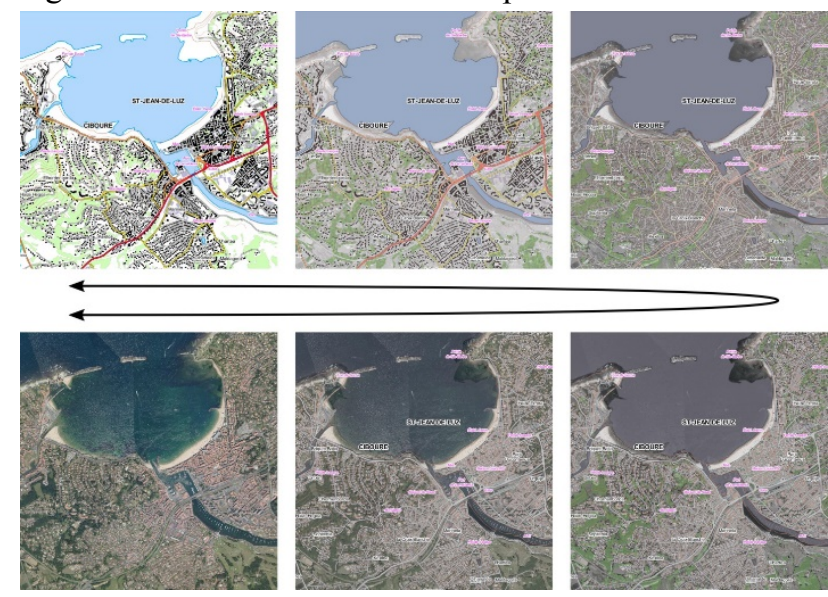

Fig. 4. Cartographic continuum between a topographic vector data with IGN-France style and an orthoimage based on method design proposed by Hoarau and Christophe (2016).

\subsection{Research challenges}

From this related works, we take up three different challenges to successfully design a cartographic continuum between two topographic styles:

1)The transitions between consecutive in-between cartographic abstractions in the continuum should be ensuring continuity and smoothness during the navigation.

2)The two original topographic styles have to be recognized throughout the navigation in the cartographic continuum.

3)The legibility of the different cartographic objects (e.g. color contrast) has to be preserved for each inbetween representation.

To reach the three research challenges, we aim at identifying which structural element of a topographic map should be interpolated and how to design them on in-between representations, in order to get a relevant and efficient cartographic continuum between two topographic styles.

\section{An exploratory approach to design a cartographic continuum between two topographic styles}

In this section, we detail our exploratory approach to design a cartographic continuum between two topographic styles: IGN-France and OSM standard Mapnik style. Our approach is declined two steps:

1)Identification of structural elements of the maps which should be interpolated.

2)Proposition of methods to design in-between representations for these structural elements. 


\subsection{A comparison method to identify the structural elements of a map which should be interpolated}

We select two topographic maps, a first one produced by the French NMA IGN-France illustrated by the Figure 5.A, a second one produced by OSM illustrated by the Figure 5.B. The two topographic maps depict the same geo-graphic context at the same scale: a French territory, St-Jean-de-Luz at the 1:25000 scale. Based on our knowledge on map design, we propose to visually compare with a systematical approach two map design processes: data se-lection and legend specifications, layer per layer, from the map A to the map B. We do not focus on other carto-graphic processes, such as data categorization or generalization for the sake of simplicity. To summarize, our approach consists in visually comparing two topographic maps, to analyze how they are designed and to identify some structural element which should be interpolated.

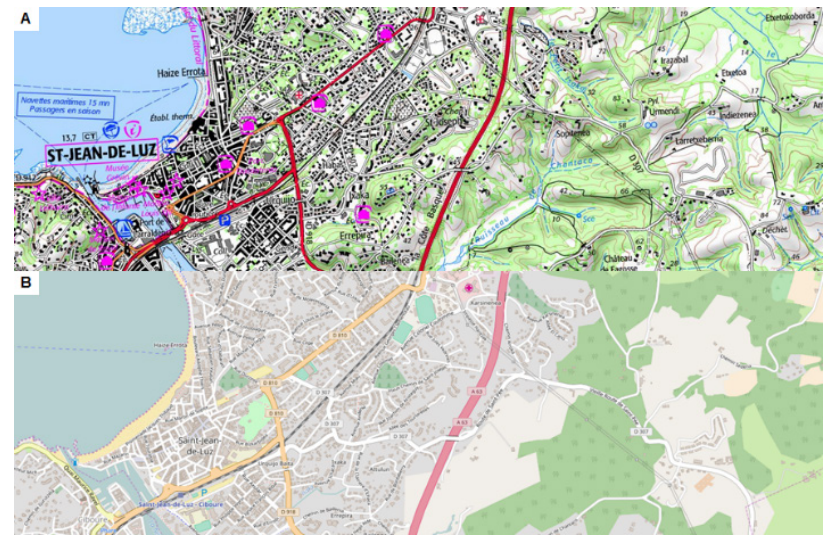

Fig. 5. Visual comparison of two topographic styles: IGNFrance (A) and OpenStreetMap (B) - COpenStreetMap contributors.

This comparison provides us a graphic detailed insight of two topographic styles. We observe particularly some differences allowing us to identify six kinds of maps structural elements should be interpolated. We group them into two categories:

\subsubsection{The data selection}

The Figure 6 illustrates the two maps structural elements, belonging data selection, identified by the visual comparison of the two topographic styles IGN-France and OpenStreetMap:

- Shading: shaded is a major cartographic theme in the IGN-France maps while is not represented in the OSM maps, as illustrated in Figure 5.

- Additional vector data: the IGN-France maps contain more cartographic themes than the OSM maps, as contour lines and the intermittent streams illustrated by the Figure 5 .

\subsubsection{The legend specifications}

The Figure 7 illustrates the four maps structural elements, belonging legend specifications, identified by the visual comparison of the two topographic styles IGN-France and OpenStreetMap:
- Color: the two maps producers use two different color palettes to design the various cartographic themes. Each color palette is illustrated on Figure 6.

- Texture: different kinds of texture are used by the two producers use to design cartographic objects belonging land use. The textures used to design forests and beaches are illustrated on Figure 6.

- Line width: the two maps producers use different specifications of line width to design the cartographic linear objects, such as roads network illustrated by Figure 5 .

- Labelling style: the two maps producers use different typography specifications to design the labels, as illustrated by Figure 5.

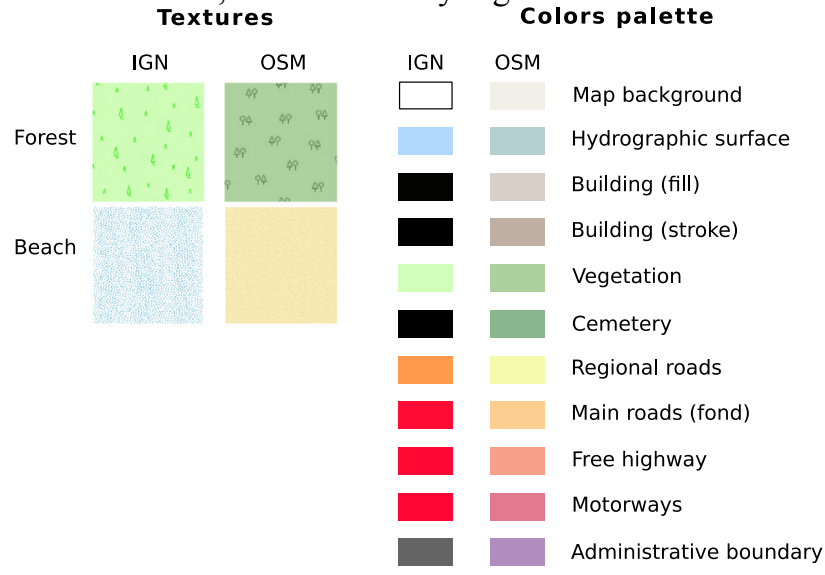

Fig. 6. Differences of texture and color specifications between IGN-France and OSM maps.

According to the first results, our approach seems relevant to identify structural elements of a map which should be interpolated. We have to notice that the results are dependent on the two topographic styles analyzed. It would be interesting to compare others topographic styles with the same approach to see if the structural elements are similar or different.

\subsection{Methods to design in-between cartographic representations}

In this section, we propose to use design methods to interpolate each structural element identified in the previous sub-section. The design methods have to comply with the research challenges briefly exposed in the subsection 2.4, namely:

1) to ensure a smooth transition all along the cartographic continuum,

2) to allow users to recognize topographic style's sources,

3) to ensure legibility of cartographic objects.

From the literature, we identified two methods allowing us to design in-between representations of a cartographic continuum:

1) A linear interpolation method: this method is used to define color specifications of each in-between cartographic representation. 
2) An arithmetic progression method: this method is used to define for each in-between cartographic represen-tation (1) line width specifications for the linear cartographic themes, (2) level of transparency of shading and (3) level of transparency of additional vector data, such as contour lines and intermittent streams.

Following the recommendation of a past research (Ory, 2015), we propose to keep the original textures and typography of the two topographic styles sources all along the cartographic continuum. Indeed, texture and typography are visual codes and guides, well known by the users, allowing them to recognize the two cartographic families of the continuum extrema. We think that keeping original textures and typography improve the user navigation be-tween the two topographic styles. In the middle of the cartographic continuum, we propose to switch sharply from the textures of style A to the textures of style B. We adopt the same approach for the typography.

\subsection{First results}

We apply the two design methods explained in the previous sub-section on 1:25000 IGN cartographic vector dataset, which depicts a French geographical space StJean-Luz. For this vector dataset, we define legend specifications of two topographic styles: IGN-France and OSM. The legend specifications are defined with the SLD/SE standard.

In this use case, we use the two design methods to interpolate color and the line width. We use the linear interpolation method to define the color specifications and we use the arithmetic method to define the line width specifications. The Figure 7 illustrates the results obtained for various cartographic themes: hydrographic surface, building, vegetation, cemetery, roads network, river stream and administrative boundary, further we add the map back-ground. In this example, we define three inbetween representations to navigate from IGN-France style to OpenStreetMap style.

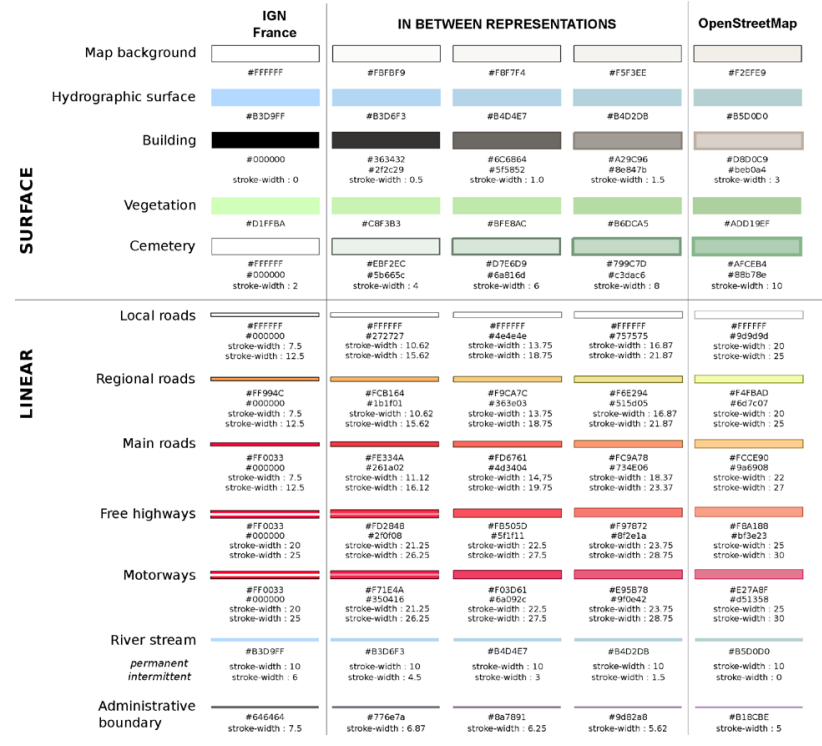

Fig. 7. Color and stroke-width specifications of surface and linear cartographic themes to interpolate IGN-France style to Open-StreetMap style.

We produce all in-between cartographic abstractions on our open source platform, GeOxygene (https://ignf.github.io/geoxygene/community/index.html).

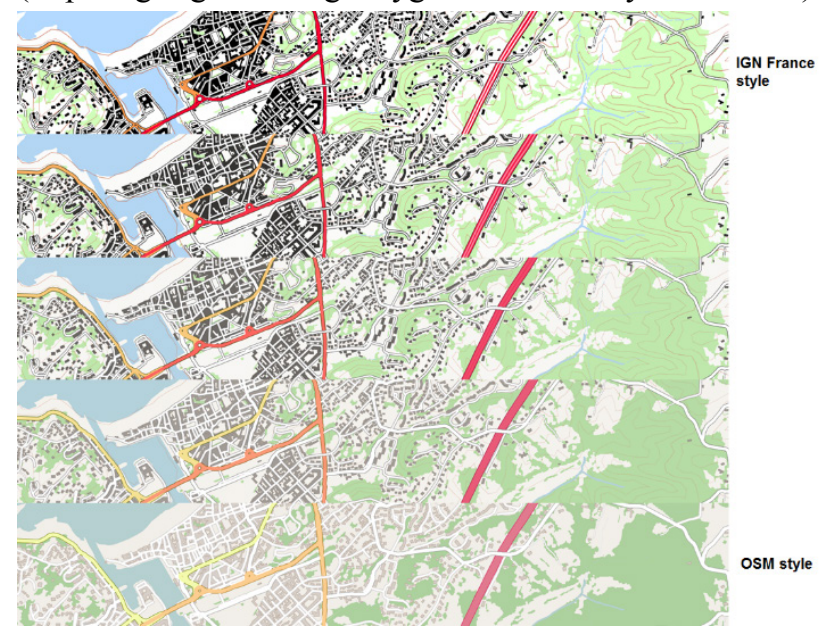

Fig. 8. Color and line width interpolation between IGN-France style and OpenStreetMap style.

The different in-between representations, illustrated by the Figure 8, constitute a cartographic continuum that allows users to have a smoothing navigation between IGNFrance style and OSM style. Indeed, the visualization of color and line-width of the different cartographic themes are progressive all along the cartographic continuum. The methods allow the resolution of two issues:

1) Navigate between two cartographic styles which displayed differently the cartographic themes: for instance, color and width differences exist between IGN-France roads network and OSM roads network. These two methods allow sliding smoothly to one style to the second one, ironing out the color and width differences.

2) Navigate between two cartographic styles with different cartographic content: for instance, the contour lines and the intermittent streams, outlined in red on Figure 8, are only displayed in the IGNFrance style. These two methods allow to progressively hide a cartographic theme and thus to iron out the content differences between the two topographic styles.

\section{Conclusion and outlook}

This paper addresses the design of cartographic continuum to navigate between two topographic abstractions with different styles. We proposed an approach to define the best legend specifications to design each in-between representation: first, we identify the major structural elements of a map which should be interpolated; then, we pro-pose two design methods to interpolate each structural element to produce in-between representation.

In future research, we hope to formalize our proposition in a framework allowing the automatic design of cartographic continuum between various topographic styles. A 
first research perspective is to measure the similarities and the difference between in-between representations to assess the quality of a cartographic continuum. A second re-search perspective issue is the improvement of color interpolation with the specification of some constraints to improve the global legibility of the map, by preserving, for instance, the color contrasts between map features. A third research perspective issue is to measure a "global distance" between two topographic styles in order to define how many in-between representations needs to design the best cartographic continuum. Finally, it will be necessary to assess the efficiency of a cartographic continuum to achieve some user visual tasks.

\section{Acknowledgements}

This work is supported by the French National Research Agency, as part of the MapMuxing project [ANR-14CE24-0011-01].

\section{References}

Beconyte, G. (2011) Cartographic styles: criteria and parameters, In Proc. 25th International Cartographic Conference (ICC'11), 3-5 July, Paris, France.

Bucher, B., Jolivet, L., Buard, E. and Ruas, A. (2007). 'The need for web legend services', in 7th International Symposium on Web and Wireless GIS (W2GIS), Lecture Notes in Computer Science, Springer ed. by Ware, J. M. and Taylor, G. E., pp. 44-60.

Christophe, S. (2012) Cartographic Styles between traditional and original (towards a cartographic style model), In proceedings of AutoCarto Conference 2012, 16-18 September, Columbus, Ohio, USA.

Dumont, M., Touya, G., Duchêne, C. (2015), Automated Generalisation of Intermediate Levels in a Multi-Scale Pyramid, In proceed-ings of 18th ICA Workshop on Generalisation and multiple Representation, August 2015, Rio de Janeiro, Brazil

Hoarau C., Christophe S. (2016). Cartographic continuum rendering based on color and texture interpolation to enhance photo-realism perception, ISPRS Journal of Photogrammetry and Remote Sensing (volume in press)

Jolivet, L. (2009) Characterizing maps to improve ondemand cartography - the example of European topographic maps, 17th Con-ference on GIScience and Research in UK (GISRUK'09), 1-3 April, Durham (UK).

Kent, A. \& Vujakovic, P. (2009) Stylistic Diversity in European State 1:50 000 Topographic Maps, The Cartographic Journal, 46(3), 179-213.

Lafay S., Braun, A., Chandler, D., Michaud, M., Ricaud, L., Mustière, S. (2015). Automatic mapping and innovative on-demand mapping services at IGN France. CaGIS - Cartography and Geographic Information Science, 42(1), 54-68.

Mericskay, B. (2011). Les SIG et la cartographie à l'ère du géoweb. L'Espace géographique, 40(2), 142-153.
Ory J., Christophe, S., Fabrikant, S.I., Bucher, B. (2015). How do map readers recognize a topographic mapping style?, The Carto-graphic Journal, 52(2), 193-203.

Ory J. (2016). Connaissances pour la conception et la perception de styles topographiques, Phd thesis in Geographic Information Sciences, Paris-Est University, IGN, LaSTIG lab., COGIT team.

Griffin, A., et Fabrikant, S.I. (2012). More maps, more users, more devices means more cartographic challenges. The Cartographic Journal, 49(4), 298-301.

Ruas, A. (2006). Usages des bases de données géographiques - exemple de l'IGN, Rencontre Jacques Cartier, Lyon, France. 